\title{
Methyl Nicotinate-Induced Vasodilation in Generalized Social Phobia
}

\author{
Martin Katzman*,1,2, Sylvie Cornacchi', Aimee Coonerty-Femiano', Bronwen Hughes', Monica Vermani', \\ Lukasz Struzik ${ }^{1,3}$ and Brian M Ross ${ }^{4}$ \\ 'Centre for Addiction and Mental Health, Toronto, Canada; ${ }^{2}$ Department of Psychiatry, University of Toronto, Toronto, Canada; ${ }^{3}$ Department of \\ Physiology, University of Toronto, Toronto, Canada; ${ }^{4}$ Highland Psychiatric Research Foundation, UHI Millenium Institute, Inverness, Scotland
}

\begin{abstract}
Elevated vasodilatory response (blushing) to social situations is characteristic of social phobia (SP). A relatively unexplored basis for this phenomenon is alteration in underlying vasodilatory mechanisms. To investigate this possibility, we evaluated the vasodilatory response induced by methyl nicotinate (niacin ester derivative) in 31 generalized SP patients and $4 \mathrm{I}$ matched healthy volunteers (HV). A patch impregnated with 0, 0.1, 0.5, I, and $10 \mathrm{~mm}$ methyl nicotinate was applied to the forearm or face of subjects for I min, followed by 20-min laser Doppler spectroscopy blood flow monitoring. Blood flow stimulation with I and $10 \mathrm{~mm}$ methyl nicotinate was significantly reduced in SP patients by 35 and 17\%, respectively. Induced blood flow was negatively correlated with patients' Leibowitz Social Phobia Scale (LSAS) at I and $10 \mathrm{~mm}$ doses. Furthermore, the maximal rate of change of vasodilatory reaction was correlated with symptom scores at I and $10 \mathrm{~mm}$ doses. Induced increases in the arm and face blood flow measurements correlated, supporting the external validity of the former location. Generalized SP patients vasodilate less to topical methyl nicotinate challenges, with effect amplification in severely ill patients. Although the mechanism for this is unclear, we propose desensitization of the prostaglandin-mediated vasodilating system as an explanation.

Neuropsychopharmacology (2003) 28, |846-185I, advance online publication, 23 July 2003; doi:I 0. I038/sj.npp. I 300227
\end{abstract}

Keywords: social phobia; vasodilation; methyl nicotinate; prostaglandins; blood flow; flux

\section{INTRODUCTION}

Social phobia (SP) has received more attention in the psychiatric literature recently, partly due to the high prevalence of this condition (13.3\% of the noninstitutionalized civilian American population) indicated in the latest National Comorbidity Study (Kessler et al, 1994). The comorbidity of this condition with other mood and anxiety disorders, its early age of onset, and chronic course make it a major contributor to psychiatric illness (Weissmann et al, 1996). Despite illness severity, the condition is often mis/ undiagnosed by physicians, often resulting in self-medication, substance abuse, frequent use of medical services, inappropriate treatments, social isolation, as well as educational and occupational impairment (Ballenger, 2000).

The diagnosis of SP is made according to the Diagnostic and Statistical Manual 4th Edition (DSM IV; American Psychiatric Association, 1994) based on marked and persistent fear of one or more social or performance

*Correspondence: Dr MA Katzman, Centre for Addiction and Mental Health, 250 College Street, Toronto, Ontario, Canada M5T IR8, Tel: + | 4169796819 , Fax: + I 4169796853

E-mail: martin_katzman@camh.net

Received 18 June 2002; revised 21 January 2003; accepted 22 January 2003

Online publication: I4 May 2003 at http://www.acnp.org/citations/ Npp05 | 402233/default.pdf situations, in which the person is exposed to unfamiliar people or possible scrutiny by others. The individual feels that they will act in a way (or show anxiety symptoms) that will be humiliating or embarrassing. Exposures to the feared social situation almost invariably provoke anxiety, which may take the form of a situational-bound or situationalpredisposed panic attack.

Perhaps most characteristic of this situational anxiety is the observation of blushing, which is a well-known (Ballenger, 2000; Fahlen, 1996; Endelmann, 1991), but also a poorly understood and inadequately studied, phenomenon (Gerlach et al, 2001; Bouwer and Stein, 1998; Stein and Bouwer, 1997; Steurer et al, 1997; Leary et al, 1992; Endelmann, 1991; Shearn et al, 1990). Furthermore, little is known about the fear of blushing and its effect on the blushing response (Gerlach et al, 2001; Mulkens and Bogels, 1999; Endelmann, 1991) or the physiology of blushing itself. Nevertheless, the severity of patient fears of blushing has resulted in the development of rather severe and invasive techniques for treatment of the disorder. One example is endoscopic transthoracic sympathicotomy (ETS), which is a surgical treatment designed to interrupt the sympathetic nerve pathways that mediate emotional facial blushing (Drott et al, 1998; Telaranta, 1998).

While it is often believed that blushing is one of the independent core features of SP, it has been suggested that 'the subjective fear of blushing' is in fact creating the 
blushing response (eg Bogels et al, 1997). This model, first elucidated by Marks and Gelder (1966) and later by Scholing and Emmelkamp (1999), described a sequence of events beginning with maladaptive cognitive processes (ie anticipatory fear of symptoms, specifically blushing) that produce distress and ultimately lead to an exacerbation of the symptoms deemed not to be under the voluntary control of the individual. This inaccurate cognitive model is further supported by Endelmann (1991) and Shearn et al (1990), who showed that the perception of blushing is initiated by the recognition of a rise in facial temperature and not by the preceding changes in blood flow or volume, which are barely detected by the individual. This perception of changes, as noted by Marks and Gelder (1966), may be what leads to the maladaptive cognitive processes (ie anticipatory fear of symptoms, specifically blushing) that produce the distress and ultimately lead to an exacerbation of the symptoms.

An opposing explanation, that the physiological mechanism of blushing is altered per se in SP, comes from a recent study using specific biological challenges that induce vasodilation. Bouwer and Stein (1998), using a similar method as that developed for patients with schizophrenia (Glen and Ward, 1999), reported that $100 \mathrm{mg}$ of oral (niacin) nicotinic acid resulted in an increased flushing response in SP compared to healthy volunteers (HV). However, Gerlach et al (2001) did not detect increased blushing induced by social stress in SP, although the perception of blushing was increased.

Given the lack of consensus on blushing in SP as well as the contrasting findings in the literature, a method to operationalize the flushing response is imperative. In order to meet this challenge, this study examined the response of generalized SP patients to a topical challenge of methyl nicotinate using a laser Doppler flow meter monitor in order to assess the flushing response in generalized SP patients vs HV numerically. An ester of nicotinic acid is used in this experiment, namely methyl nicotinate, due to the latter's better ability to penetrate the skin (Glen and Ward, 1999). In addition, the methodology used is particularly suitable for human use, given that common confounding variables such as nicotine (cigarette) and caffeine (coffee, tea) use have no effect upon nicotinic acid response when used chronically (Turenne et al, 2001).

\section{METHODS}

\section{Subjects}

A total of 31 generalized SP patients and $41(\mathrm{HV})$ were recruited via advertisement at the Centre for Addiction and Mental Health (Table 1). Participant eligibility was determined using a structured clinical interview (SCID) as per DSM-IV criteria, with diagnosis confirmation via a standard psychiatric consultation for the SP patients. Patients were diagnosed with generalized SP if they reported anxiety or distress judged by the interviewer to be at least moderate in a minimum of three situations listed in the Anxiety Disorders Interview Schedule IV (ADIS-IV; Di Nardo et al, 1994) and if at least three different situational domains (eg formal speaking and interaction, informal speaking and interaction, observation of behavior, and assertive interac-
Table I Subject details

\begin{tabular}{lccccc}
\hline Group & N & Age & Sex & BP (mmHg) & LSAS \\
\hline Healthy volunteers & 41 & $32 \pm 2$ & $20 \mathrm{M} / 2 \mathrm{IF}$ & $126 / 70$ & - \\
Social phobia & 31 & $36 \pm 2$ & $13 \mathrm{M} / / 8 \mathrm{~F}$ & $121 / 70$ & $62 \pm 5$ \\
\hline
\end{tabular}

Age and LSAS are shown as mean \pm SEM. BP: blood pressure; LSAS: Liebowitz Social Anxiety Scale. The ratio of males to females did not differ significantly between HV and SP groups $\left(\chi^{2}\right.$-test; $\left.P>0.05\right)$, nor did age or blood pressure ( $t$ test; $P>0.05$ ).

tion) were represented in this list of situations. For those subjects who completed it (21 of 31 patients), the Liebowitz Social Anxiety Scale (LSAS; Heimberg et al, 1999) was used as a measure of severity of SP symptoms. All SP patients voluntarily participated prior to the initiation of clinical treatment. All subjects were considered competent to consent and provided written informed consent after a full explanation of the research protocol. Testing lasted approximately $2 \mathrm{~h}$ and participants received nominal compensation for their time and travel expenses (as required by ethical protocol consideration). Exclusion criteria included: comorbidity with other Axis I diagnoses within 6 months prior to study enrollment, treatment by any traditional or alternative pharmacotherapy, coexisting major medical illness, allergic reactions to aspirin or analgesics, hypotension, hypertension/high cholesterol treated with (oral dose) nicotinic acid or niacin, and inflammatory or chronic pain conditions, including those treated with regular use of nonsteroidal anti-inflammatory drugs (NSAIDs).

\section{Procedure}

After eligibility was established and prior to application of each methyl nicotinate patch, a 5-mm diameter circle of relatively hairless, unblemished skin was masked off with hypoallergenic medical tape. This area was located on either the subjects' inner forearm or face (centre of cheekbone-below the outside corner of the eye). Initially, a 5-min baseline laser Doppler measurement was taken. Subsequently, the niacin patch was applied over the same skin area on the forearm $(0,0.1,0.5,1$, or $10 \mathrm{~mm})$ or face $(1 \mathrm{~mm}$ only) for $1 \mathrm{~min}$ and removed. This was followed by a 20 -min period during which blood flow was measured continuously. All 31 SP patients and all $41 \mathrm{HV}$ received all methyl nicotinate doses in a fixed order fashion (lowest-highest dose) each on the same skin location. There was no dosecompounding effect of methyl nicotinate from one dose application to the next within this 20-min interpatch interval. In those subjects in which both face and arm blood flows were measured, the 1-mm dose applied to the arm was run simultaneous to that on the face. A total of 11 SP patients and $11 \mathrm{HV}$ subjects received the methyl nicotinate patch on both the face and the forearm.

Vasodilation was induced using the methyl ester derivative of niacin (methyl nicotinate) in patch (percutaneous) formulations of $0,0.1,0.5,1$, or $10 \mathrm{~mm}$ drug. The doses were attained from a standard $1 \mathrm{M}$ methyl nicotinate stock solution that is then diluted with water to the desired doses. Vasodilation was measured by using a Moor Instruments moorLAB laser Doppler spectroscope adjusted to measure 
changes in cutaneous blood flow. Laser Doppler flowmetry takes real-time measurements of local tissue blood flow by reflecting light off a moving object, such as a red blood cell. Each measurement is assigned an arbitrary unit (Flux) that is a parameter based on the summed intensity of reflections recorded by the instrument (Moor Instruments Limited, 1998). Flux was sampled 40 times per second and transferred to a personal computer for analysis.

\section{Curve Fitting}

Blood flow (flux) data were averaged into 30 -s bins and analyzed using nonlinear curve fitting (Prism, Graphpad software). The flux at any time $t(F(t))$ is fitted to a curve with parameters $F(0)$, the baseline flux, $\Delta F$, the maximal plateau change in flux (ie change in blood flow), $\mu$, the maximal rate of change, and $L$, the lag time between application of the test patch and occurrence of vasodilation (see model Figure 1). Runs that did not produce a significant reaction (this being defined as at least one block of five consecutive 30-s time periods being significantly (Student's $t$-test; $P<0.05$ ) above baseline blood flow) were assigned a $\Delta F$ value of zero; the $\mu$ and $L$ parameters could not be calculated in these circumstances.

\section{Statistical Analysis}

Fitted parameters were compared between HV and SP groups at each dose using the nonparametric MannWhitney $U$-test, since some of the parameters did not meet the Shapiro-Wilk test for normality. Linear regressional analysis was performed by computing the Spearman correlation coefficient when symptom-rating scales were involved, and the Pearson correlation coefficient in other cases. To eliminate the potential of gender and age as confounds, the analysis included a comparison of fitted parameters between sexes with fitted parameter correlation to the age of SP and HV groups.

\section{RESULTS}

Baseline blood flow did not differ significantly (MannWhitney $U ; P>0.05$ ) between SP and HV groups at any dose (Table 2). As the dose of methyl nicotinate was increased, the proportion of patients producing a significant elevation in blood flow also increased, although this proportion did not differ significantly between SP and HV groups (Table 2). The distribution of maximal change in blood flow $(\Delta F)$ values was found to differ significantly from normality at the 1 and $10 \mathrm{~mm}$ methyl nicotinate doses in both SP and HV groups (Shapiro-Wilk; $P<0.05$ ), necessitating the use of nonparametric statistics for subsequent analyses. Median $\Delta F$ values were significantly (Mann-Whitney $U$-test; $P<0.05$ ) decreased in the SP compared to HV group at both the 1 and $10 \mathrm{~mm}$ doses by 35 and $17 \%$, respectively (Table 2).

In runs in which significant blood flow increases were detected, the maximal rate of change $(\mu)$ and lag time between drug application and blood flow commencing $(L)$ were also calculated (Table 2). Neither was found to differ significantly between the SP and HV groups (MannWhitney $U ; P>0.05)$. None of the calculated parameters differed significantly between male and female subjects (Mann-Whitney $U ; P>0.05$ ), with the exception of a significantly $(P=0.046)$ higher $\mu$ parameter in female SP subjects for the 10- mM dose of methyl nicotinate only. Age did not significantly $(P>0.05)$ correlate with any parameter, except for $\mu$ at the 10-mm dose in the SP group (Pearson's correlation coefficient $=-0.39 ; P=0.029$ ).

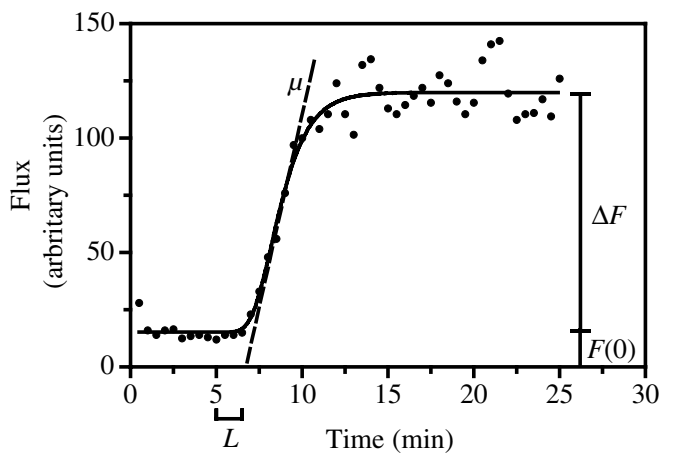

Figure I Modeling of blood flow data. Following a 5-min baseline blood flow measurement using laser Doppler flowmetry, methyl nicotinate (I mM) was applied to the forearm of a healthy volunteer at time $=5 \mathrm{~min}$. The patch was removed I min later and blood flow measurements were resumed. Data points are flux values averaged over $30 \mathrm{~min}$. Data were then fitted to an equation using nonlinear curve fitting to give baseline flow $(F(0))$, maximal plateau phase increase in blood flow $(\Delta F)$, the lag time between patch application and the start of vasodilation $(L)$, and the maximal rate of change in blood flow ( $\mu$, the gradient of the dashed line), as illustrated.

Table 2 Vasodilatory changes induced by methyl nicotinate applied to the forearm of healthy volunteers and patients with social phobia

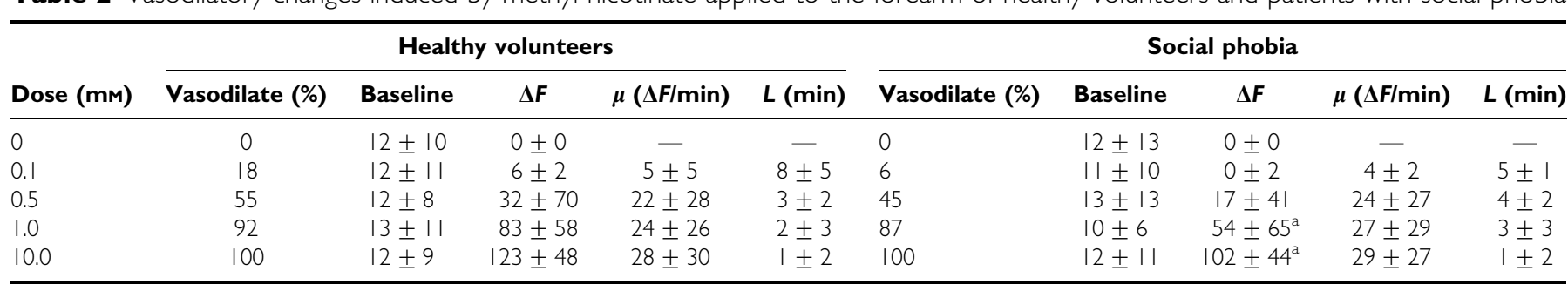

Methyl nicotinate or saline was applied to the forearms of $4 \mathrm{I}$ health volunteers and 31 patients with social phobia. The percentage of patients who react to the drug (vasodilate) are shown along with baseline blood flow in arbitrary flux units. The maximal plateau change in blood flow $(\Delta F)$, maximal rate of change $(\mu)$, and the time between drug application and the start of the reaction $(L)$ are median \pm interquartile range. Note that $\mu$ and $L$ are only calculated in those cases in which a significant reaction to the drug is observed.

${ }^{a} P<0.05$ vs healthy volunteers by Mann-Whitney U-test. 


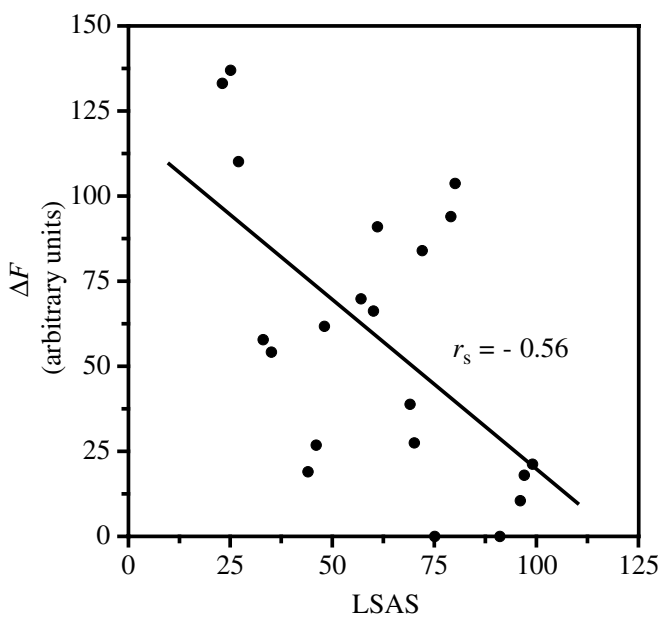

Figure 2 Relationship between LSAS and methyl nicotinate-induced vasodilation. Methyl nicotinate (I mM) was applied to the forearms of 21 patients with SP. Maximal change in blood flow $(\Delta F)$ is shown plotted against LSAS scores. The best-fit line is shown along with the Spearman correlation coefficient.

In a subgroup of SP subjects $(n=21)$, an assessment was undertaken to examine the relationship between the LSAS score and the nature of vasodilation. $\Delta F$ was significantly negatively correlated with the LSAS score at the 1 and $10 \mathrm{~mm}$ methyl nicotinate dose (Spearman correlation coefficients were -0.56 and -0.50 , respectively; $P<0.05$ ) (Figure 2), as was $\mu$ at the same doses (Spearman correlation coefficients were -0.58 and -0.50 , respectively).

Finally, the vasodilatory effect of $1 \mathrm{~mm}$ methyl nicotinate on the arm was simultaneously compared to that on the face in a subgroup of $11 \mathrm{SP}$ patients. Although baseline blood flow was considerably higher on the face $($ median $=58.3$ ) than the arm (median $=15.4)$, both $\Delta F$ and $\mu$ parameters were highly correlated between the two application points (Pearson's correlation coefficients were 0.79 and 0.80 , respectively) (Figure 3 ).

\section{DISCUSSION}

The major finding of our study was that of a significant difference in the vasodilatory response to topical methyl nicotinate in SP compared to HV patients. This was not noted at all doses of methyl nicotinate, which may be due to a relatively low number of subjects showing any significant reaction at the low doses in both SP and control groups.

The inverse correlation between the vasodilatory response and the LSAS scores suggests that the greater the severity of patients' condition, the less likely they are to vasodilate. While our finding that decreased vasodilation is most pronounced in the most severely ill patients appears counterintuitive, this may be accounted for by the nature of disease progression.

One may account for this initially confusing finding by noting that assessment of our subjects' vasodilatory response took place in their adulthood, but that SP itself begins in childhood. In a group of 63 participants, Fahlen (1996) found the median age of onset for SP was 14.5 years with $95 \%$ of patients studied, indicating a sharp increase in

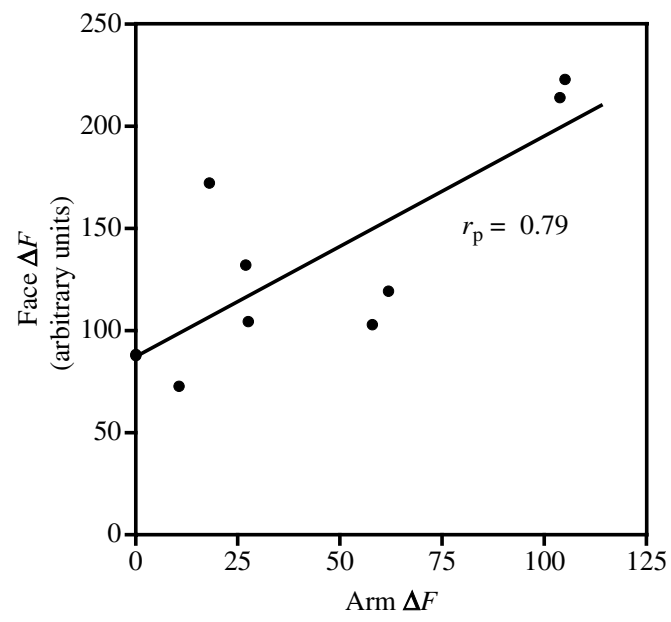

(arbitrary units)

Figure 3 Relationship between arm and facial vasodilation induced by methyl nicotinate. Methyl nicotinate (I mM) was applied simultaneously to the forearm or face of I I patients with SP. Maximal change in blood flow $(\Delta F)$ at each application site is shown plotted against the other. The best-fit line is shown along with the Pearson correlation coefficient.

insecurity with social contacts after the age of 10 years. Perhaps then there is a downregulation in the intensity of the vasodilatory response in SP after chronic exposure to blushing/vasodilation. If correct, this suggests that earlystage SP patients may blush more than chronic cases, a possibility that could explain the lack of consensus in the literature as to whether socially phobic patients are more likely to blush (eg Marks and Gelder, 1966; Bogels et al, 1997; Scholing and Emmelkamp, 1999; Shearn et al, 1990; Endelmann, 1991; Gerlach et al, 2001 vs Bouwer and Stein, 1998). Alternatively, reduced vasodilatory response to niacin may be an intrinsic feature of persons who develop more severe SP symptoms. While this again may seem counterintuitive, the possibility should be considered that the heightened perception of blushing, rather than increased blushing magnitude per se, reported by some investigators, may relate to the patient having a normally low vasodilatory response to certain physiological stimuli. Against a lowered background vasodilatory theater, patients may have a relatively larger perceived blush response when in socially stressful situations than that in healthy controls. It would be of interest therefore to examine flushing stimulated by other stimuli in social phobics, such as heat and transient skin ischemia, to determine if they mirror the blunted response observed using niacin. Lastly, though unlikely, it is possible that methyl nicotinate-induced changes in blood flow may be altogether unrelated to the underlying pathophysiology of SP, as the drug's effects may be mediated by as yet unknown and unrelated mechanisms.

Since we collected vasodilatory data from patients' arms, it could therefore be argued that the vasodilatory reaction in the arm is not equivalent to an area in which blushing is normally observed, namely the face. We have partially addressed this potential problem by demonstrating a high degree of correlation between the vasodilatory responses of the face and arm (see Figure 3), suggesting that data obtained using the arm can be generalized to the face. This is of importance given that it is simpler to obtain data, 
especially when using multiple doses of methyl nicotinate, from the arm than the face.

Our data showing decreased vasodilation (flushing) in the $\mathrm{SP}$ relative to the $\mathrm{HV}$ groups are in direct contrast to earlier findings of increased flushing in SP patients in response to oral nicotinic acid administration (Bouwer and Stein, 1998). Indeed, since SP patients exhibit flushing as a prominent symptom of disease presentation, one may have expected them to exhibit increased vasodilation in response to a stimulus. Although possible, it is unlikely that this difference is accounted for by differences in stimulus delivery, since both topical and oral nicotinic acid/methyl nicotinate administration reliably provokes flushing in healthy subjects as evidenced by this and Bouwer and Stein's work. Furthermore, the rather unpleasant nature of oral vs topical nicotinic acid administration (eg uncomfortable feelings of heat, hearing and feeling blood flow, feeling faint due to induced hypotension) may have overstimulated endogenous blushing mechanisms that are more related to tolerance for aversive experiences and less to directly related SP mechanisms. Further attempts to reconcile the two studies require consideration of the mechanism of the flushing response and possible differences between SP patients and controls in that mechanism.

The mechanism underlying decreased topical methyl nicotinate response in SP is presently unclear. However, it has now been well established that both methyl nicotinate and nicotinic acid-induced flushing response is the result of increased prostaglandin synthesis in skin cells, suggested to be macrophages or adipocytes, which then act upon capillary epithelial cells to cause vasodilation (Wilkin et al, 1982, 1985; Morrow et al, 1989; Turenne et al, 2001). Interestingly, healthy subjects develop tolerance to the nicotinic acid-induced flushing response (Stern et al, 1991). This appears to be due to diminished prostaglandin $D_{2}$ synthesis, which has been implicated as the main prostaglandin subtype responsible for nicotinic acid-induced vasodilation (Morrow et al, 1989). Thus, a likely explanation for our findings of decreased methyl nicotinate-induced flushing in the SP population is downregulation of the prostaglandin synthetic pathway. One can conceive that a population exposed to repeated bouts of flushing via repeated activation of a biosynthetic pathway (PG synthesis pathway) would, over time, downregulate that pathway's activity. The details of how such downregulation may come about in the SP population or in $\mathrm{HV}$ remain to be elucidated. Furthermore, it is uncertain how niacin mediates the release of PGs and where in the biosynthetic pathway of PGs this downregulation of synthesis takes place (eg at the level of the phospholipid, its release from membrane via phospholipase A2 activity, cyclic adenosine monophosphate turnover, G-protein activity, etc). Alternatively, altered niacin response in SP may reside in a desensitization of the PG target, the capillary epithelial cells themselves. Tupler et al's (1997) work also suggested phospholipid involvement in SP pathology using magnetic resonance spectroscopy studies, although a precise framework of the biochemical linkage involving prostaglandins as specific culprits was not suggested.

Interestingly, decreased methyl nicotinate response has also been observed in schizophrenia (Glen and Ward, 1999), although the magnitude of the reduction is much greater in schizophrenia than in SP. Nevertheless, the presumed involvement of phospholipid-associated signaling systems in methyl nicotinate desensitization in schizophrenia, as well as the novel treatment approaches currently being tested to treat schizophrenia (Rybakowski and Weterle, 1991; Hudson et al, 1997; Fenton et al, 2000) suggest that similar strategies may be useful in SP.

Although PG synthesis downregulation or capillary desensitization in SP patients seems the most likely mechanisms accounting for the results presented here, the hypothesis goes beyond the data presented. Future work in the biochemistry of SP should include measurement of plasma PG levels in SP vs HV, as well as other agents that may be involved, including tryptophan and serotonin turnover.

As a final note, it should be noted that the conclusions drawn apply to the generalized subtype of SP. Others have found differences in physiological arousal as measured by heart rate during behavioral tests between generalized and nongeneralized SP (Heimberg et al, 1990; Levin et al, 1993; Hofmann et al, 1997; Boone et al, 1999). It is possible that such differences in responsiveness extend to the flushing response between SP subtypes.

\section{CONCLUSION}

Thus, our data, in contrast to those of Bouwer and Stein (1998), show that SP patients appear to exhibit a reduction in vasodilatory response to topically applied methyl nicotinate (on the arm) in comparison to HV. PG synthesis downregulation seems to be the most likely explanation for this reduced response in SP patients. Further studies are warranted to understand the underlying biochemical mechanism, for example measurement of nicotinic acidinduced release of prostaglandins and other eicosanoids in SP. In addition, the apparent correlation of methyl nicotinate-induced vasodilatation with symptom severity in SP suggests that further studies are warranted that should investigate whether nicotinate challenge is predictive of outcome or is useful as a method of assessing treatment modalities.

\section{ACKNOWLEDGEMENTS}

This research was funded by the Research in Psychiatry Grants Program of the Centre for Addiction and Mental Health.

\section{REFERENCES}

American Psychiatric Association (1994). Diagnostic and Statistical Manual of Mental Disorders, 4th edn. American Psychiatric Press: Washington, DC.

Ballenger JC (2000). Recognizing the patient with social anxiety disorder. Int Clin Psychopharmacol 1: S1-S5.

Bogels SM, Mulkens S, De Jong PJ (1997). Task concentration training and fear of blushing. Clin Psychol Psychother 4: 251-258.

Boone ML, McNeil DW, Masia CL, Turk CL, Carter LE, Ries BJ et al (1999). Multimodal comparisons of social phobia subtypes and avoidant personality disorder. J Anxiety Disord 13: 271-292. 
Bouwer C, Stein DJ (1998). Hyperresponsivity to nicotinic acid challenge in generalized social phobia: a pilot study. Eur Neuropsychopharmacol 8: 311-313.

Di Nardo PA, Brown TA, Barlow DH (1994). Anxiety Disorders Interview Schedule IV for DSM-IV-lifetime. Graywind Publications: Albany, NY.

Drott C, Claes G, Olsson-Rex L, Dalman P, Fahlen T, Gothberg G (1998). Successful treatment of facial blushing by endoscopic transthoracic sympathicotomy. Br J Dermatol 138: 639-643.

Endelmann RJ (1991). Correlates of chronic blushing. Br J Clin Psychol 30: 177-178.

Fahlen T (1996). Core symptom pattern of social phobia. Depress Anxiety 4: 223-232.

Fenton WS, Hibbeln J, Knable M (2000). Essential fatty acids, lipid membrane abnormalities, and the diagnosis and treatment of schizophrenia. Biol Psychiatry 47: 8-21.

Gerlach AL, Wilhelm FH, Gruber K, Roth WT (2001). Blushing and physiological arousability in social phobia. J Abnorm Psychol 110: $247-258$.

Glen I, Ward PE (1999). Oral and topical niacin flush testing in schizophrenia. In: Peet M, Glen I, Horrobin D (eds). Phospholipid Spectrum Disorder in Psychiatry. Marius Press: UK. pp 139-144.

Heimberg RG, Hope DA, Dodge CS, Becker RE (1990). DSM-III-R subtypes of social phobia: comparisons of generalized social phobics and public speaking phobics. J Nerv Mental Dis 178: 172-179.

Heimberg RG, Horner KJ, Juster HR, Safren SA, Brown EJ, Schneier FR et al (1999). Psychometric properties of the Liebowitz Social Anxiety Scale. Psychol Med 29: 199-212.

Hofmann SG, Newman MG, Ehlers A, Roth WT (1997). Speech disturbances and gaze behavior during public speaking in subtypes of social phobia. J Anxiety Disord 11: 573-585.

Hudson CJ, Lin A, Cogan S, Cashman F, Warsh JJ (1997). The niacin challenge test: clinical manifestation of altered transmembrane signal transduction in schizophrenia? Biol Psychiatry 41: 507-513.

Kessler RC, McGonagle KA, Zhao S, Nelson CB, Hughes M, Eshleman $S$ et al (1994). Lifetime and 12-month prevalence of DSM-III-R psychiatric disorders in the United States: results from the National Comorbidity Survey. Arch Gen Psychiatry 51: 8-19.

Leary MR, Britt TW, Cutlipp II WD, Templeton JL (1992). Social blushing. Psychol Bull 112: 446-460.

Levin AP, Saoud JB, Strauman T, Gorman JM, Fyer AJ, Crawford R et al (1993). Response of 'generalized' and 'discrete' social phobics during public speaking. J Anxiety Disord 7: 207-221.

Marks I, Gelder MG (1966). Different ages of onset in varieties of phobias. Am J Psychiatry 123: 218-221.

Moor Instruments Limited (1998). Moor Instruments floLAB/ moorLAB Laser Doppler Blood Flow Monitor User Manual, Section 1.611.

Morrow JD, Parsons III WG, Roberts II LJ (1989). Release of markedly increased quantities of prostaglandin D2 in vivo in humans following the administration of nicotinic acid. Prostaglandins 38: 263-274.

Mulkens S, Bogels SM (1999). Learning history in fear of blushing. Behav Res Ther 37: 1159-1167.

Rybakowski J, Weterle R (1991). Niacin test in schizophrenia and affective illness. Biol Psychiatry 29: 834-836.

Scholing A, Emmelkamp PM (1999). Prediction of treatment outcome in social phobia: cross-validation. Behav Res Ther 37: 659-670.

Shearn D, Bergman E, Hill K, Abel A, Hinds L (1990). Facial coloration and temperature responses in blushing. Psychophysiology 27: 687-693.

Stein DJ, Bouwer C (1997). Blushing and social phobia: a neuroethological speculation. Med Hypotheses 49: 101-108.

Stern RH, Spence JD, Freeman DJ, Parbtani A (1991). Tolerance to nicotinic acid flushing. Clin Pharmacol Ther 50: 66-70.

Steurer J, Hoffmann U, Bollinger A (1997). Skin hyperemia in a habitual blusher. Vasa 26: 135-136.

Telaranta T (1998). Treatment of social phobia by endoscopic thoracic sympathicotomy. Eur J Surg 5(Suppl 580): 27-32.

Tupler LA, Davidson JR, Smith RD, Lazeyras F, Charles HC, Krishnan KR (1997). A repeat proton magnetic resonance spectroscopy study in social phobia. Biol Psychiatry 42: 419-424.

Turenne SD, Seeman M, Ross BM (2001). An animal model of nicotinic-acid-induced vasodilation: effect of haloperidol, caffeine and nicotine upon nicotinic acid response. Schizophr Res 50: 191-197.

Weissmann MM, Bland RC, Canino GJ, Greenwald S, Lee CK, Newman SC et al (1996). The cross-national epidemiology of social phobia: a preliminary report. Int Clin Psychopharmacol 1: $15-23$.

Wilkin JK, Fortner G, Reinhardt LA, Vogt Flowers O, Kilpatrick J et al (1985). Prostaglandins and nicotinate-provoked increase in cutaneous blood flow. Clin Pharmacol Ther 38: 273-277.

Wilkin JK, Wilkin O, Kapp R, Donachie R, Chernosky ME, Buckner J (1982). Aspirin blocks nicotinic acid-induced flushing. Clin Pharmacol Ther 31: 478-482. 